\title{
The Contingency Theory of Management as a Factor of Acknowledging the Leaders-Managers of Our Time Study Case: The Practice of the Contingency Theory in the Company Avrios
}

\author{
Blerona Shala, Abetare Prebreza*, Berim Ramosaj \\ University of Pristina "Hasan Pristina”, Faculty of Economics, Prishtina, Kosovo \\ Email: ^abetare.prebreza@gmail.com
}

How to cite this paper: Shala, B., Prebreza, A. and Ramosaj, B. (2021) The Contingency Theory of Management as a Factor of Acknowledging the Leaders-Managers of Our Time Study Case: The Practice of the Contingency Theory in the Company Avrios. Open Access Library Journal, 8: e7850. https://doi.org/10.4236/oalib.1107850

Received: August 10, 2021

Accepted: August 31, 2021

Published: September 3, 2021

Copyright $\odot 2021$ by author(s) and Open Access Library Inc.

This work is licensed under the Creative Commons Attribution International License (CC BY 4.0).

http://creativecommons.org/licenses/by/4.0/

\begin{abstract}
The purpose of this paper is to find leaders-managers of different styles who work in the same company, have similar tasks but different jobs and to distinguish between them in terms of success, work achieved in relation to others and work in general. The main objectives of this paper are: (1) analyzing the connection between the style and the situation of the leader as the main point in a certain context; (2) using the contingent approach model to find the leader style and adapt the style to the given situation; (3) drawing conclusions about the importance of proper placement of the leader where he fits best to have as much benefit as possible. The secondary goal will be the analysis, will be to increase the effectiveness of these leaders-managers in their work. This paper aims to understand the importance of placing the Leader in the best location or position for greater profit. The main goal of all leaders-managers is to lead the followers towards a common goal. The paper will discuss Avrios, a company focused on managing car software that we will be discussed later.
\end{abstract}

\section{Subject Areas \\ Management, Leadership, Contingency \\ Keywords \\ Business Theories, Human Resource Management, Organizational Behavior}




\section{Theoretical Framework}

The contingency theory of Management is a Leaders-Managerial-Adaptation theory, which means that the Leader tries to adapt to different situations [1].

It is called contingent because it suggests that a leader's effectiveness depends on how well his/her style fits the context. This approach tells us that a leader, or the way a leader leads in one environment, is different in another. Fred Fiedler is known as the person who has elaborated the theory of contingency in more detail, so this will be the author who will elaborate more on this topic as he is the most known and respected worldwide.

The essence of Fiedler's theory is that the effectiveness of a leader depends on a combination of two forces: the Leader leaders-managerial style, and the favored situation. One of the essential problems when it comes to the success of a company is the perfect position of the leader in the context where it fits the most, for example in the case when the company is focused on increasing production where the work is technically a leader focused on achieving the goal will design any plan that can lead there without necessarily taking into account the relationships of employees or their wishes, on the other hand if a company is working to increase teamwork for a project where you definitely need collaboration then a leader more focused on getting the consent of the workers then the leader with such a personality would fit better.

\subsection{The Contingency Approach Theory}

Fred Fiedler is of Austrian descent; he is one of the leading scholars in the field of leadership and organizational performance. Fred Fiedler is the founder of contingency theory that shows the relationship between leadership effectiveness and situational circumstances. Fielder argued in the 1960s that the approach of effective leadership depends on the contingencies of the situation, demanding the nature of the task and how secure they are.

The Contingency Theory of Leadership was developed by Fred Fiedler in 1958 during his research on the effectiveness of the leader in group situations. Fiedler believed that his effectiveness in leading depends on controlling the situation and the style of Leadership.

The research on leadership by the author and his collaborators, developed since 1951 [2], has led to a theory of Leadership called the "Contingency Model". This theory lays out the contingent relationship between leadership performance based on a framework of leadership style points, called the "Least Preferred Coworker scale", or LPC. This relationship is moderated by a "favoritism" dimension which is conceptually defined as the degree to which leadership, whose situation enables the leader to control and influence his/her group behavior.

The theory is that leaders with low LPC scores (describing their less-favored peers in a very negative way) work better in very favorable and relatively unfavorable situations; leaders with high LPC scores (describing their peers in relatively positive terms) work best in situations which are intermediate in favor. 
The theoretical model, first introduced in 1964, has been validated with some modifications in a large number of subsequent studies. The LPC score has been used in numerous investigations on interpersonal relationships, problems in education, employee satisfaction, the art of leadership clearly represents an important predictor in interpersonal behavior.

Fred Fiedler is a theorist whose Contingent Approach was the forerunner of contingency management theory. Fiedler believed he had a direct connection to the traits of a leader and the effectiveness of a Leader. According to Fiedler, some traits of leadership helped in a certain crisis and so leadership would have to change given the new circumstances.

Fiedler's Theory of Contingency proposes the following concepts:

1. Fiedler's Contingency Theory says there is no better way to manage an organization.

2. Fiedler's contingency theory says that leadership theory says that a leader should be able to identify which management style will help achieve the goals of the organization in a particular situation.

3. The main component of Fiedler's contingency theory is the most preferred co-worker scale (LPC) which measures a manager's leadership orientation.

Organization classification according to 3 variables according to Fred Fiedler contingent approach:

The 3 variables to consider are: how well employees perceive the leader, the degree to which the employee's jobs are described in detail, and the authority the leader possesses through his/her position in your organization. Contingency theories claim that leaders will be able to exert more influence if they are able to have good relationships with employees. In addition, contingency management states that in organizations where the task is described in detail, the leader has more influence over employees than in those organizations that do not have structured tasks.

Fiedler's Contingency Theory says that there are many internal and external factors that can affect the optimal organizational structure. These factors include the size of the organization, the technology that is in use, the leadership style, and how the organization can adapt to changes in strategy.

\subsection{Contingency Approach, Key Factors: Style and Situation}

Fred Fiedler created contingency theory by studying the styles of many different leaders working in different contexts, primary military organizations. He praised the leaders' styles, the situation in which they worked and whether they were effective. After analyzing the styles of hundreds of leaders, they were both good and bad, Fiedler and his colleagues were able to make empirical generalizations based on which leadership styles were best and which styles were worst, for a given organizational context.

In February, contingency theory is related to different styles and situations. Provides the framework to effectively match the leader to the situation. 


\subsubsection{The Contingency Approach Key Factor: Style}

Within this structure to adapt the leader to the styles envisaged in this approach, we divide these as follows:

- Task motivated,

- Relationship motivated [3].

Work-motivated leaders are primarily concerned with achieving a goal, while leaders motivated by maintaining relationships with others are associated with developing close interpersonal relationships. To measure Leadership style, Fiedler developed a measurement framework for measuring the least preferred collaborator to work with. Leaders who score high on this scale are described as motivated relationships and those who score low are identified as motivated tasks. The name of the scale is LPC-Least Preferred Co-Worker Framework.

The LPC scale is used in forecasting theory to measure a person's leadership style. For example, it measures your style by instructing you to describe an associate with whom you have had difficulty completing a job. The person chosen should not be a person you do not like or like personally, but a person you would prefer to work with less. Once you have selected this person, the LPC tool asks you to describe your associate in 18 sets of adjectives. Then add the points and finally we have the result that in the following we will explain what it means.

Results can be: Low LPCs, Medium LPCs, High LPCs.

Low LPCs are task motivated. Primary needs are to complete tasks, and their secondary needs are focused on dealing with people. In a work environment, they are concerned about achieving success in certain tasks, even at the cost of poor interpersonal relationships with co-workers. Some LPCs gain self-confidence by achieving their goals. They pursue interpersonal relationships, but only after first directing them toward the tasks of a group.

Middle LPCs are independent leaders in society. In the context of work, they drain themselves and do not deal much with the task or the way they see others. They are more distant from the situation and operate more independently than low or high LPCs.

High LPCs are motivated by maintaining good relationships with others. These people derive their great satisfaction in an organization from interpersonal relationships. A High LPC sees positive qualities even in the associate she or he prefers the least, even though a high LPC does not work well with that person. An organizational environment, senior LPC participates in tasks, but only after he or she is confident that relationships between people are in good shape.

The end result of the LPC is the sum of the numbers you have in the Framework. If the score is 57 or below, you are a low LPC, which suggests you are motivated towards the task. If your score is within the range of 58 to 63 , you are a medium LPC, which means you are independent. People who score 64 or above are called high LPCs and are thought to be more motivated by the relationships they have with others.

Because LPC is a personality measure, the result obtained on the LPC scale is 
believed to be left consistent over time and does not change easily. Few LPCs tend to remain high. Research shows that the reliability of test and remake of LPC test is very strong [4].

\subsubsection{The Contingency Approach Key Factor: Situation}

Contingency theory suggests that sites can be characterized by three factors:

- Leader-member-follower relations

- Task structure and

- Position strength [1]

Leading member relationships consist of group atmosphere and the ability of trust, loyalty and attraction that followers feel for the Leader. If the group atmosphere is positive and subordinates believe as well as unite with their leader, the leader-member relationship is defined as good. On the other hand, if the atmosphere is unfriendly and there is friction within the group, the leader-member relationship is defined as weak.

The second variable of the situation, the structure of the task, is the degree to which the requirements of a task are clear and written. Statements that are fully structured tend to give the leader more control, while vague and inaccurate tasks reduce the leader's control and influence.

The task is considered completed when:

(a) The job requirement is clean, stated and recognized by the people required for them finished them,

(b) The way to accomplish the task has few alternatives,

(c) The completion of the task can all be demonstrated,

(d) There are only a limited number of correct solutions to the task [2].

An example of a highly structured task is cleaning the milkshake machine at McDonald's. The rules for doing it are clearly shown to the employees, there is only one way to do it, if it is done it can be verified and if it is done properly it can be easily determined. For example, a highly unstructured task is the task of running a fundraiser for a local voluntary organization.

Doing a fundraiser has no clear rules to follow, there are many alternative ways to do it, the accuracy of how the funds came in cannot be verified, and there is no single better way to do the fundraiser funds.

The power of position, the third characteristic of situations, is the authority of the amount that a leader must reward or punish the followers. It includes the authority of the amount that a leader should reward or punish followers. It includes individuals in legitimate power who benefit as a result of position power in an organization. The power of power is strong if a person has the authority to hire and give rise in rank or pay; it is weak if a leader has no right to do these things.

The contingency theory predicts that certain styles are effective in certain situations. People who are motivated by the task (low LPC score) will be effective in both very favorable and very unfavorable situations, i.e. in situations that go 
together, those that are out of control. People who are motivated by relationships (high LPC score) are effective in favorable positions, i.e. in situations where there is a degree of security, but things are not completely under their control nor out of control.

By measuring the leader's LPC score and the three situation variables, you can predict whether the leader will be effective in a given environment. The relationship between leader style and different types of situations is illustrated in Figure 1. The figure is best understood by interpreting the rows from top to bottom. For example, a situation that has good leadership relationships, a structured task, and a strong positive power, would fall into category 6 leadership style. The assessment of the three situations can place each organizational context into one of eight categories in the following Figure.

Once the nature of the investigation is determined, the fit between the Leader's style and the situation can be assessed. The figure shows that low LPCs are effective in Categories 1, 2, 3 and 8, while high LPCs are in effect in Categories 4, 5, 6 and 7. Medium LPCs are effective in Categories 1,2, and 3. If the leader style matches the right category in the middle, he or she will be effective; if the leader's style does not match the category, he or she will not be effective.

It is important to note that contingency theory states that Leaders are not effective in all situations. If your style is not effective in all situations. If your style is a good match for the situation in which you work, you will be good at work; if your style does not match the situation, you will probably fail.

The categories of the low LPSs and medium LPC are the same, regarding the good relation of leaders and members. These are proven categorizations of the scale of freed fiddler. This is how he put the middle LPCs results of the ones participating in this questionnaire. Medium LPCs need the relations between leaders and members. To be good in order to properly bring out the best on their potential.

Fiedler's Contingency Model: An Eight-Octant Continuum

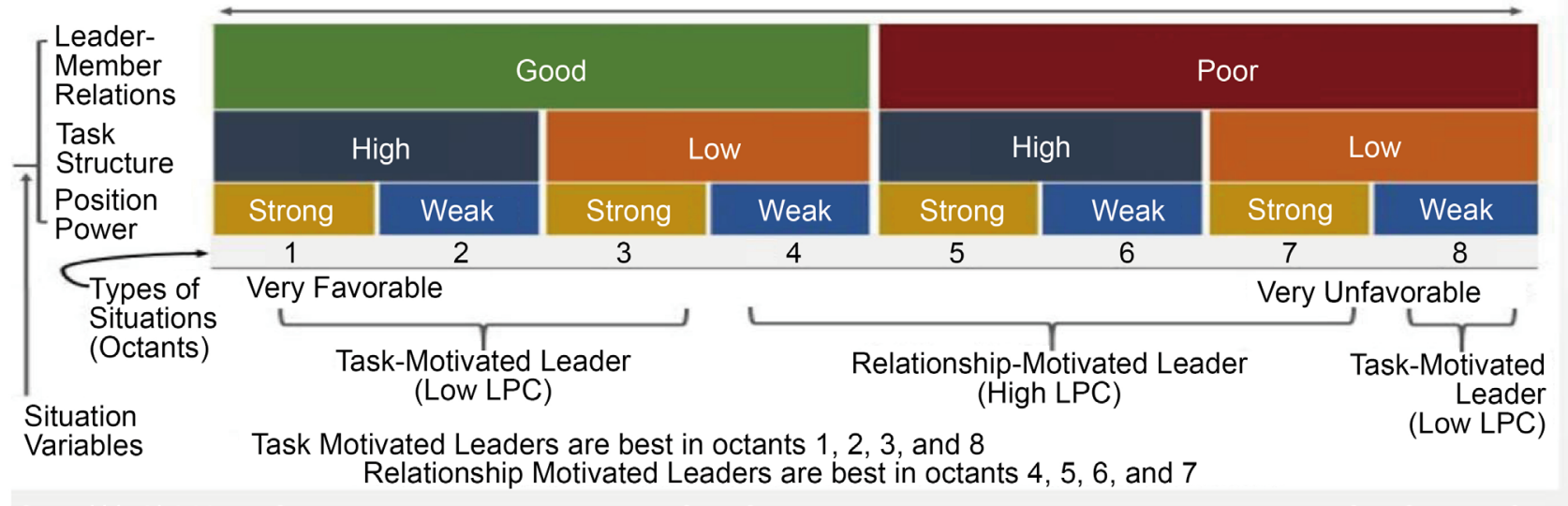

Figure 1. Contingency approach model. Source: adapted from: A Theory of Leadership Effectiveness, by F.E Fiedler, New York, (1967) [2]. 


\subsection{The Contingency Approach Advantages and Disadvantages}

\subsubsection{The Contingency Approach: Advantages}

Contingency theory has several major advantages. It is first supported by a number of empirical studies such as Peter, Hartke, \& Pohlman in 1985; Strube \& Garcia in 1981. In an era in which popular news accounts of "how to be a successful leader", contingency theory offers an approach to leadership that has a long tradition. Many studies have tested it and found it to be a valid and reliable approach to explaining how effective leadership can be achieved. Contingency theory is based on research. Below in Table 1, are shown the most advantages of the contingency approach.

\subsubsection{The Contingency Approach: Disadvantages}

Although many studies support the validity of contingency theory, it has received much criticism in the research literature. A brief discussion of these criticisms will help clarify the overall value of contingency theory as a leadership theory. Below in Table 2, are shown the most disadvantages of the contingency approach.

\subsection{The Application of the Contingency Approach}

Contingency theory has many applications in the organizational world: it can be used to answer a range of questions about the leadership of individuals in

Table 1. Advantages of the contingency approach.

\begin{tabular}{cc}
\hline No. & Advantages of The Contingency approach \\
\hline 1 & A valid and reliable approach to explaining how effective leadership can be achieved \\
2 & Is based on research \\
4 & Provides useful information about the type of leadership that is most likely to be effec- \\
5 & Useful to organizations in developing leadership profiles \\
6 & Develop profiles on individuals to determine how and where they would best serve an \\
\end{tabular}

Table 2. Disadvantages of the contingency approach.

\begin{tabular}{|c|c|}
\hline No. & Disadvantages of The Contingency approach \\
\hline 1 & $\begin{array}{l}\text { Failing to fully explain why people with certain leadership styles are more effective in } \\
\text { some situations than in others }\end{array}$ \\
\hline 2 & Focus so much on relationships that they fail to do a task \\
\hline 3 & $\begin{array}{l}\text { Difficult for respondents to understand how their descriptions of another person on } \\
\text { the scale reflect their leadership style }\end{array}$ \\
\hline 4 & Problematic to measure \\
\hline 5 & The scale instructions are not clear \\
\hline 6 & Often challenged \\
\hline
\end{tabular}


different types of organizations. For example, it can be used to explain why a person is in an ineffective position even though the person is a conscientious, loyal and hardworking manager. Also, theory can be used to predict whether a person who has worked well in a position in an organization will be just as effective if moving to a completely different position in the same company. Furthermore, contingency theory may indicate changes that senior management may wish to make in a lower-level position in order to guarantee a good fit between an existing manager and a particular work context. These are just some of the ways in which this theory can be applied in organizational settings.

\section{Empirical Results on the Contingent Approach-Case of the Study Avrios Company}

Avrios Company has a total of 272 employees in the following departments: Product Development, Marketing, Sales, Customer Service and Ceo. The questionnaires were attended by a total of 74 people employed in Kosovo, divided into two teams of the Marketing and Sales department located in Pristina, called Invoice-Team and Prospekt-Team. The questionnaire was distributed to all employees who did not hesitate to answer the questions posed.

Invoice-Team. This team is related to the financial part of the sales department, where customer invoices are processed and the gross and net cost of the car fleet of companies that use the system for effective cost reduction is calculated, for this team calculates the current costs around leasing, re-principles, maintenance services, taxes, traffic fines, etc.

Prospekt-Team. This team has the primary task of contacting a new potential customer, the fleet managers in their company and thus the growth of the firm and the companies that use this platform.

\subsection{Graphic Results from the Questionnaire from of the Scale LPC-Least Preferred Co-Worker Scale}

Below are graphically the results for the 18 questions in the Contingent Access questionnaire (Figures 2-19).

In the first question with 74 respondents, there are 11 persons who have printed 1, 14.9\%, 12 persons who have printed 2, 16.2\%, 14 persons who have printed 3, 18.9\%, 7 persons who have printed 4, 9.5\%, 7 people who typed 5 so $9.5 \%, 2$ people who typed 6 so $2.7 \%, 10$ people who typed 7 so $13.5 \%$ and 11 people who typed 8 so $14.9 \%$. The largest number turns out to be the click of number, while the lowest click turns out to be number 6 in this case.

In the second question with 74 respondents, there are 4 persons who have printed 1, 5.4\%, 9 persons who have printed 2, 12.2\%, 14 persons who have printed 3, 18.9\%, 12 persons who have printed $4,16.2 \%, 14$ people who typed 5 $18.9 \%, 5$ people who typed 6 so $6.8 \%, 5$ people who typed 7 so $10.8 \%$ and 8 people who typed 8 so $10.8 \%$. The largest number turns out to be the click of the number 3 and 5, while the lowest click turns out to be number 1 in this case. 


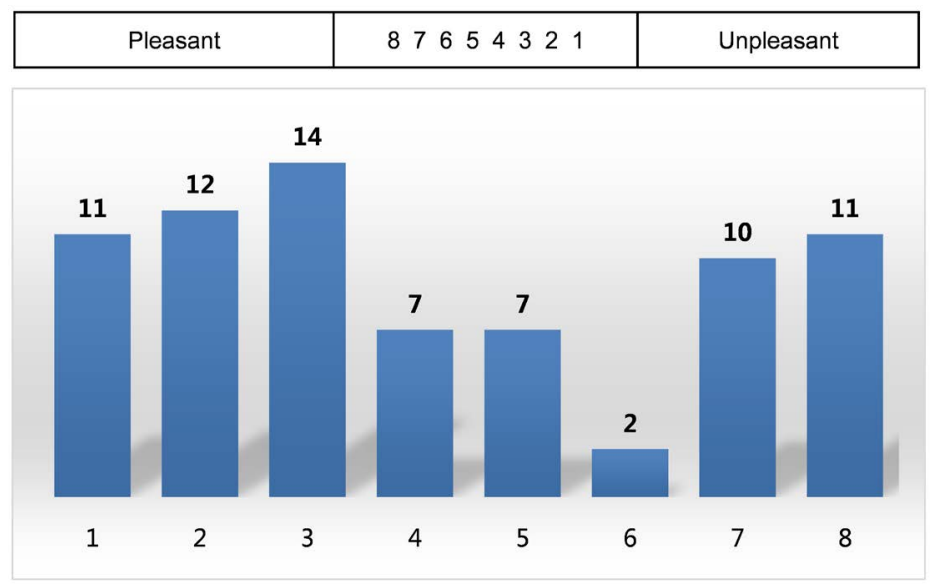

Figure 2. Presentation of the results for the first question from the contingency approach questionnaire. Source: adapted from “The LPC Questionnaire," in improving Leadership Effectiveness [2].

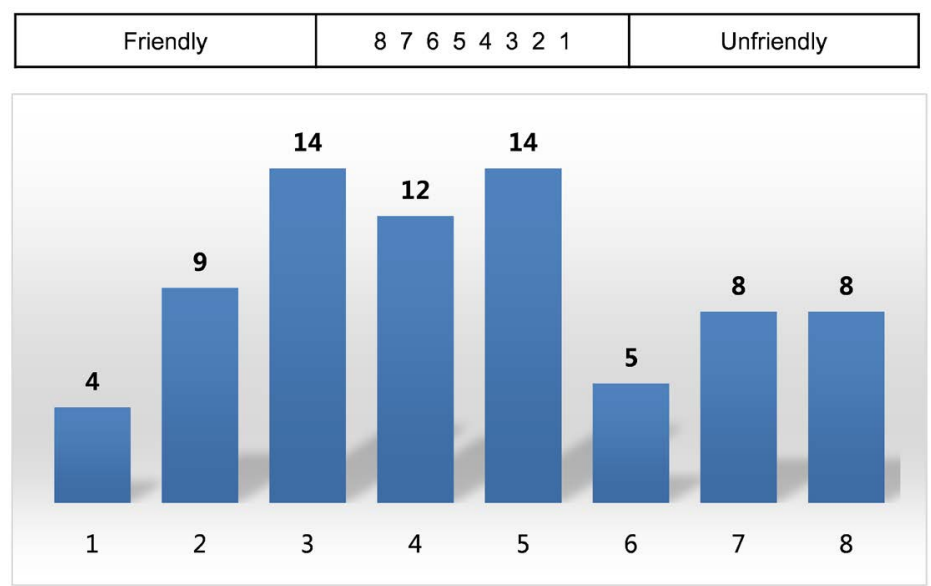

Figure 3. Presentation of the results for the second question from the contingency approach questionnaire. Source: adapted from “The LPC Questionnaire," in improving Leadership Effectiveness [2].

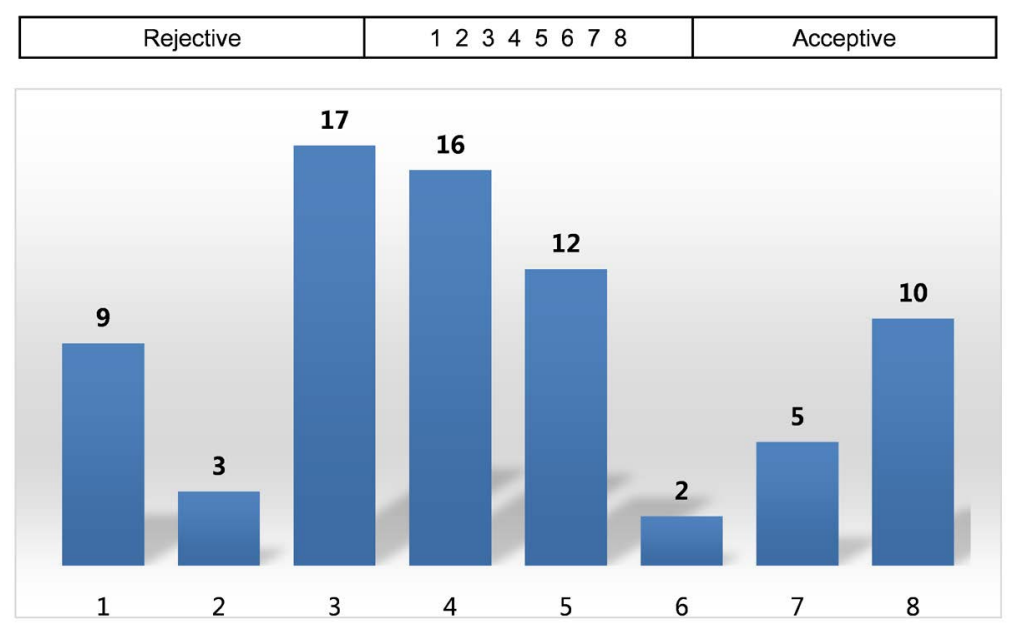

Figure 4. Presentation of results for the third question from the contingency approach questionnaire. 
In the third question with 74 respondents, there are 9 persons who have printed 1, 12.2\%, 3 persons who have printed 2, 4.1\%, 17 persons who have printed 3, 23\%, 16 persons who have printed 4, 21.6\%, 12 people who typed 5 so $16.2 \%, 2$ people who typed 6 so $2.7 \%, 5$ people who typed 7 so $6.8 \%$ and finally 10 people who typed 8 so $13.5 \%$. The largest number turns out to be click the number 3, while the lowest click turns out to be the number 2 in this case.

In the fourth question with 74 persons surveyed, there are 5 persons who have printed 1, 6.8\%, 9 persons who have printed 2, 12.2\%, 14 persons who have printed $3,18.9 \%, 9$ persons who have printed $4,12.22 \%, 12$ people who typed 5 so $16.2 \%$, 8 people who typed 6 so $10.8 \%$, 6 people who typed 7 so $8.1 \%$ and finally 11 people who typed 8 so $14.9 \%$. The largest number turns out to be click the number 3 , while the lowest click turns out to be the number 1 in this case.

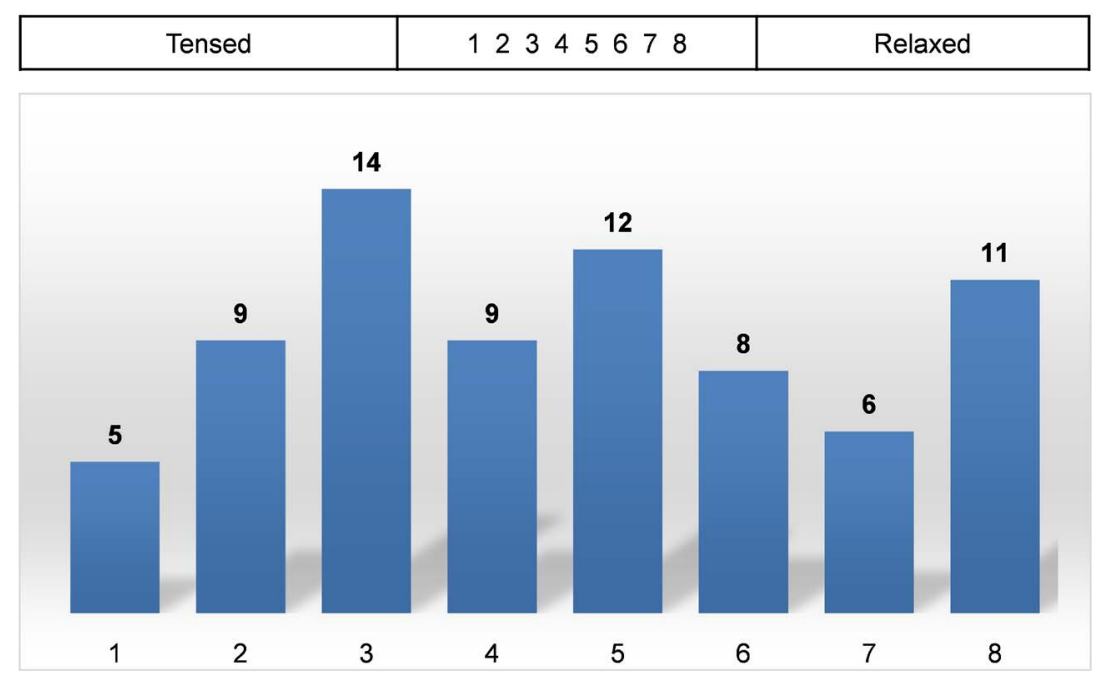

Figure 5. Presentation of the results for the fourth question from the contingency approach questionnaire.

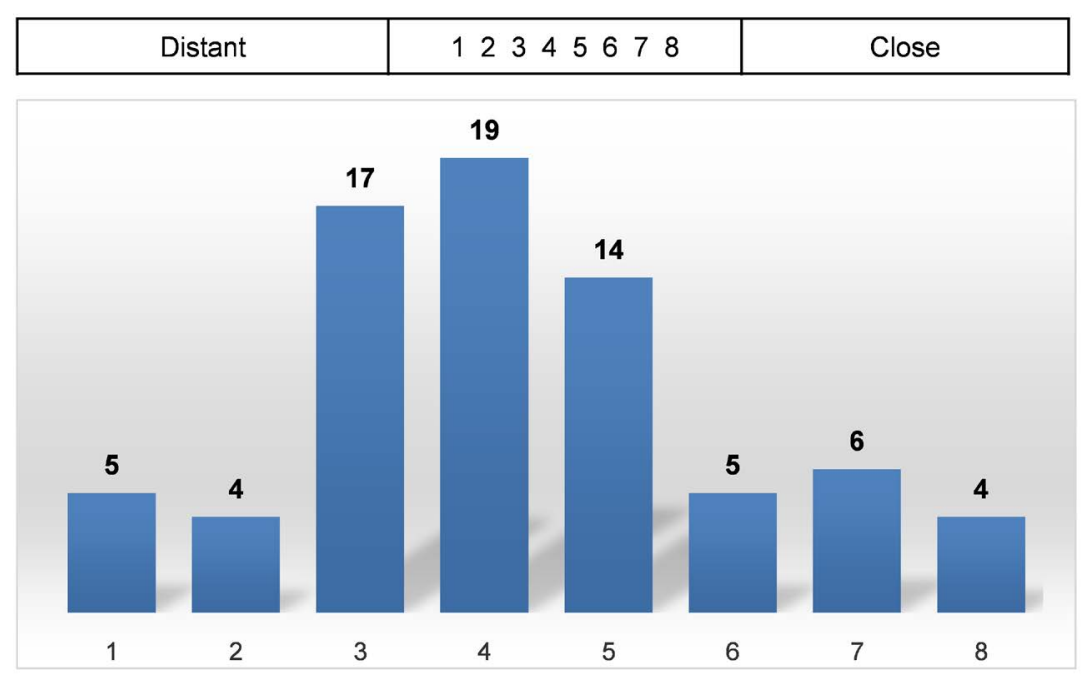

Figure 6. Presentation of results for the fifth question from the contingency approach questionnaire. 
In the fifth question with 74 persons surveyed, there are 5 persons who have printed 1, 6.8\%, 4 persons who have printed 2, 5.4\%, 17 persons who have printed 3, 23\%, 19 persons who have printed 4, 25.7\%, 14 people who typed 5 so $18.9 \%, 5$ people who typed 6 so $6.8 \%, 6$ people who typed 7 so $8.1 \%$ and finally 4 people who typed 8 so $5.4 \%$. The largest number turns out to be click the number 4 , while the lowest click turns out to be the number 2 and 4 in this case.

In the sixth question with 74 respondents, there are 4 persons who have printed 1, 5.4\%, 9 persons who have printed 2, 12.2\%, 8 persons who have printed 3 , $10.8 \%, 18$ persons who have printed $4,24.3 \%, 17$ people who typed 5 so $23 \%, 4$ people who typed 6 so $5.4 \%, 10$ people who typed 7 so $13.5 \%$ and finally 4 people who typed 8 so $5.4 \%$. The largest number turns out to be click the number 4 , while the lowest click turns out to be the number 1 and 6 in this case.

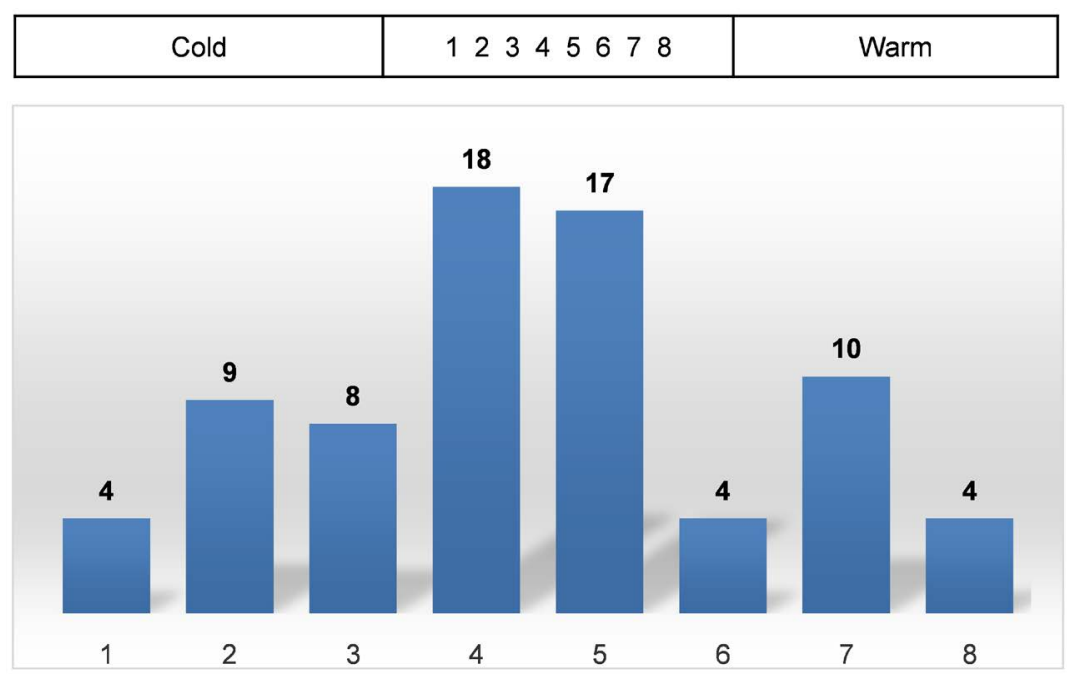

Figure 7. Presentation of results for question six from the contingency approach questionnaire.

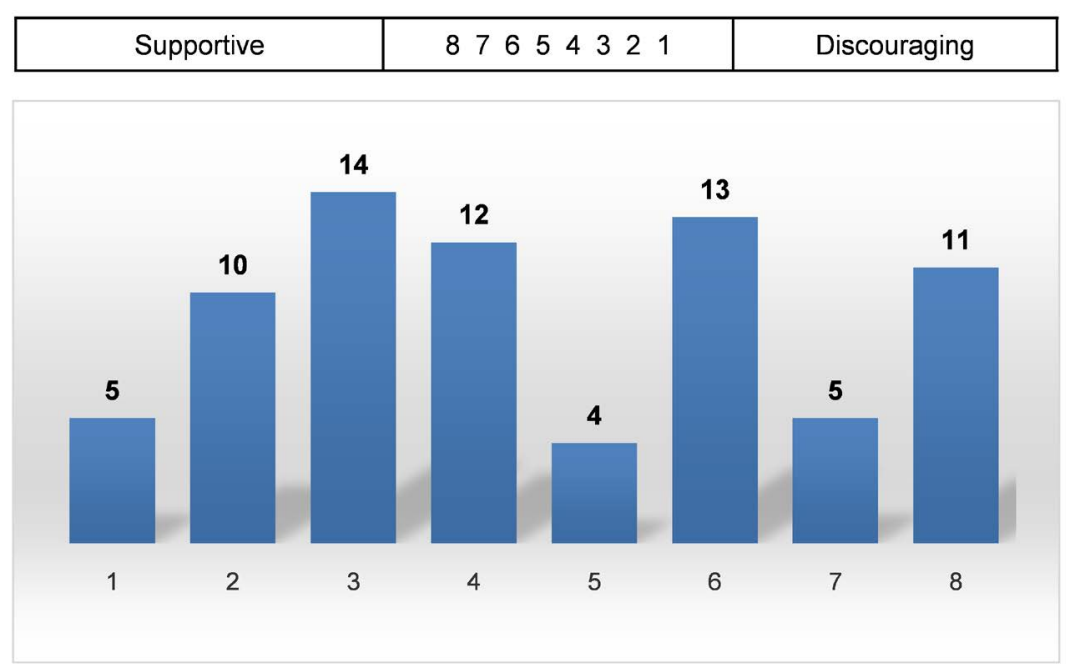

Figure 8. Presentation of the results for the seventh question from the contingency approach questionnaire. 
In the seventh question with 74 respondents, there are 5 persons who have printed 1, 6.8\%, 10 persons who have printed 2, 13.5\%, 14 persons who have printed 3, 18.9\%, 12 persons who have printed 4, 16.2\%, 4 persons who have printed 5 so $5.4 \%, 13$ persons who have printed 6 so $17.6 \%$, 5 persons who have printed 7 so $6.8 \%$ and finally 11 persons who have printed 8 so $14.9 \%$. The largest number turns out to be the click of the number 3, while the lowest click turns out to be the number 5 in this case.

In the eighth question with 74 respondents result in 1 person who typed 1 so $1.4 \%, 8$ people who typed 2 so $10.8 \%, 10$ people who typed 3 so $13.5 \%, 13$ people who typed 4 so $17.6 \%$, 18 people who typed $24.3 \%$, 10 people who typed 6 so $13.5 \%, 5$ people who typed 7 so $6.8 \%$ and finally 9 people who typed 8 so $12.2 \%$. The largest number turns out to be the click of number 5 , while the lowest click turns out to be number 1 in this case.

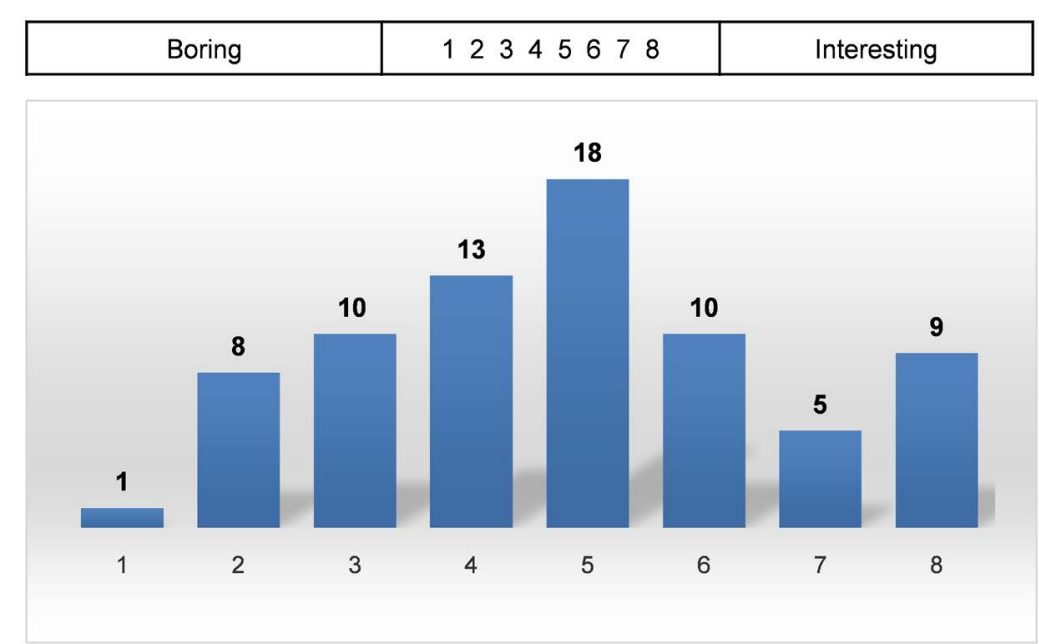

Figure 9. Presentation of results for question eight from the contingency approach questionnaire.

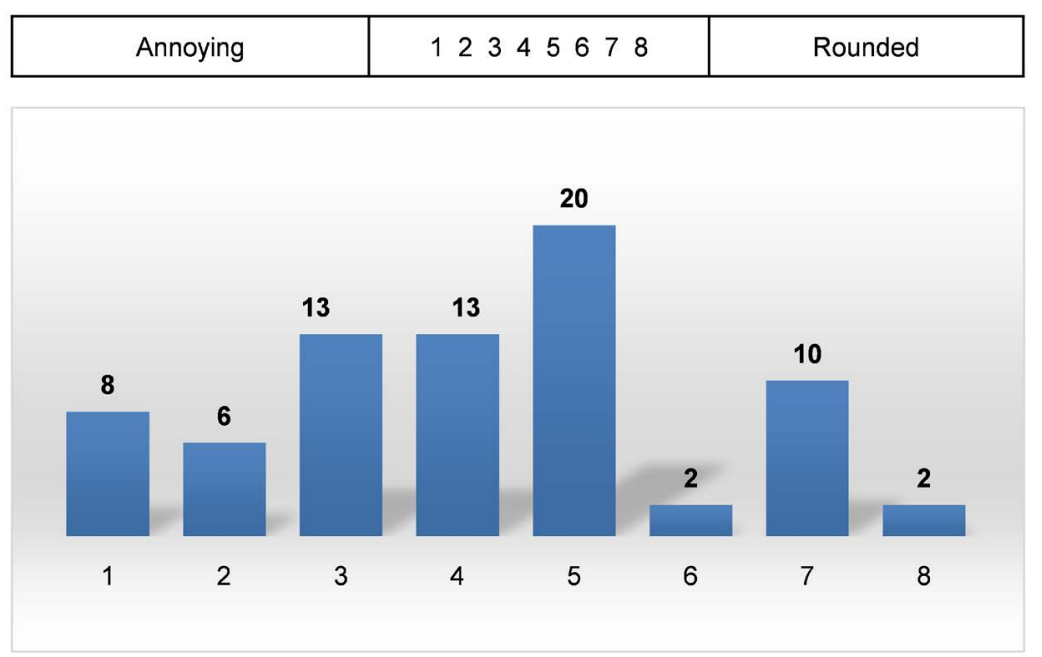

Figure 10. Presentation of the results for the ninth question from the contingency approach questionnaire. 
In the ninth question with 74 respondents, there are 8 persons who have typed $1,10.8 \%, 6$ persons who have typed $2,8.1 \%, 13$ persons who have typed $3,17.6 \%$, 13 persons who have typed $4,17.6 \%, 20$ people who typed 5 so $27 \%$, 2 people who typed 6 so $2.7 \% 10$ people who typed 7 so $13.5 \%$, and finally 2 people who typed 8 so 2.27 . The largest number turns out to be the click of the number 5 , while the lowest click turns out to be the number 6 and 8 in this case.

In the tenth question with 74 respondents, there are 4 persons who have printed 1, 5.4\%, 3 persons who have printed 2, 4.1\%, 10 persons who have printed 3, 13.5\%, 19 persons who have printed 4, 25.7\%, 18 people who typed 5 so $24.3 \%$, 8 people who typed 6 so $10.8 \%$, 9 people who typed 7 so $12.2 \%$ and finally 3 people who typed 8 so $4.1 \%$. The largest number turns out to be click the number 4 , while the lowest click turns out to be the number 2 and 8 in this case.

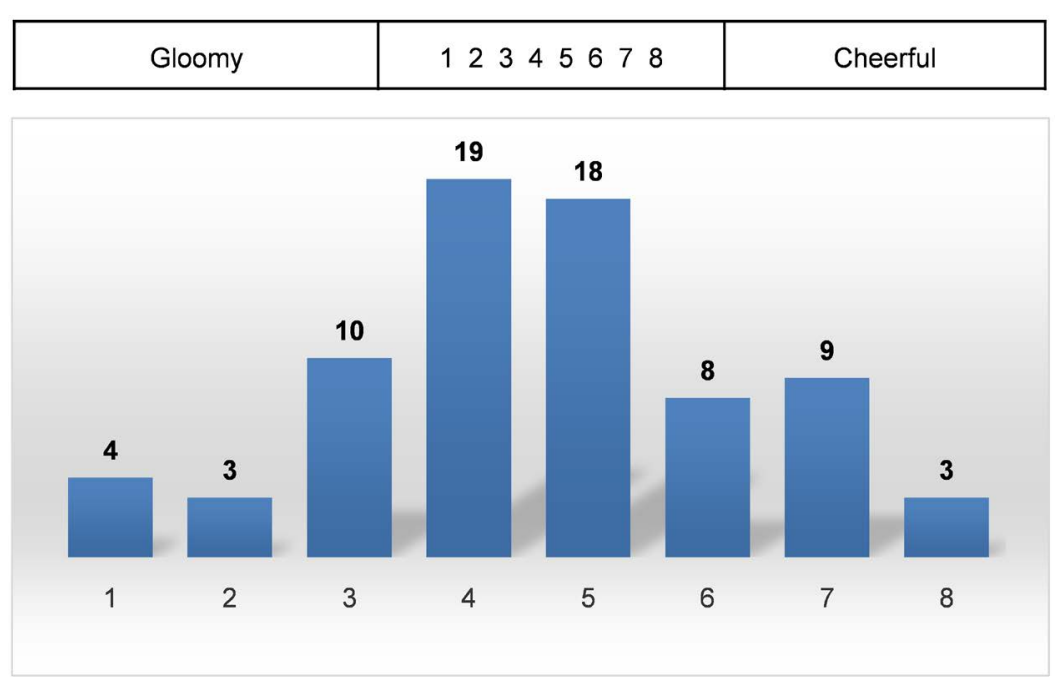

Figure 11. Presentation of results for question ten from the contingency approach questionnaire.

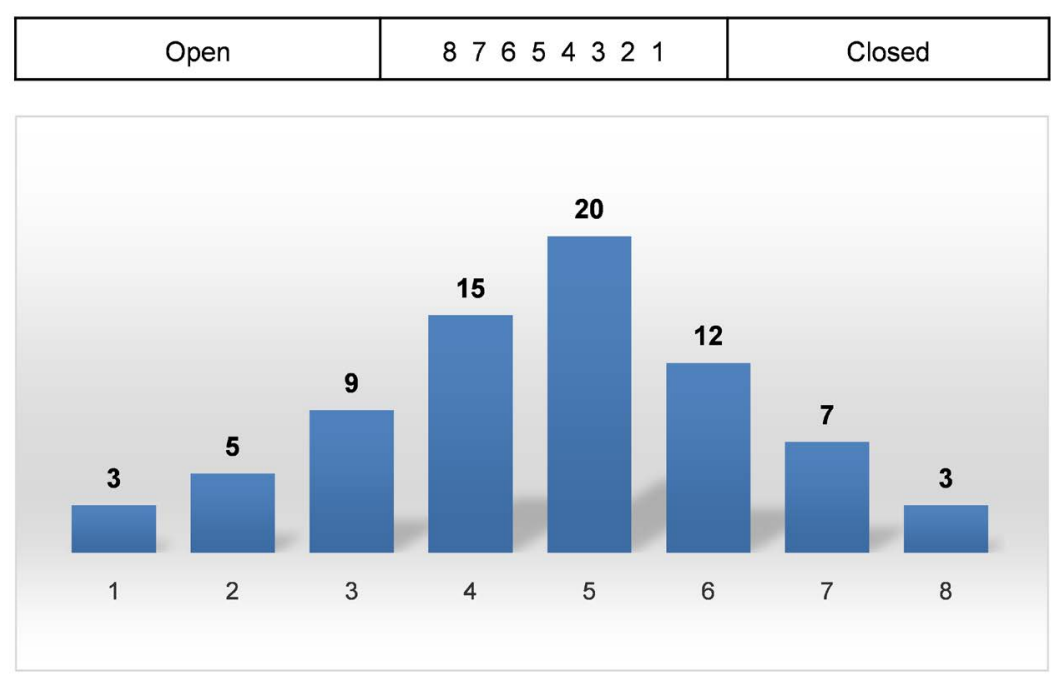

Figure 12. Presentation of the results for question eleven from the contingency approach questionnaire. 
In the eleventh question with 74 respondents, there are 3 persons who typed 1 , $4.1 \%, 5$ persons who typed $2,6.8 \%, 9$ persons who typed $3,12.2 \%, 15$ persons who typed 4, 20.3\%, 20 people who typed 5 so $27 \%, 12$ people who typed 6 so $16.2 \%, 7$ people who typed 7 so $9.5 \%$, and 3 people who typed 8 so $4.1 \%$. The largest number turns out to be the click of number 5 , while the lowest click turns out to be number 1 and 8 in this case.

In the twelfth question with 74 respondents, there are 4 persons who have printed 1,5.4\%, 6 persons who have printed 2, 8.1\%, 12 persons who have printed $3,16.2 \%, 11$ persons who have printed $4,14.9 \%, 18$ people who typed 5 so $24.3 \%, 7$ people who typed $9.5 \%, 10$ people who typed 7 so $13.5 \%$, and finally 6 people who typed 8 so $8.1 \%$. The largest number turns out to be the click of number 5 , while the lowest click turns out to be number 1 in this case.

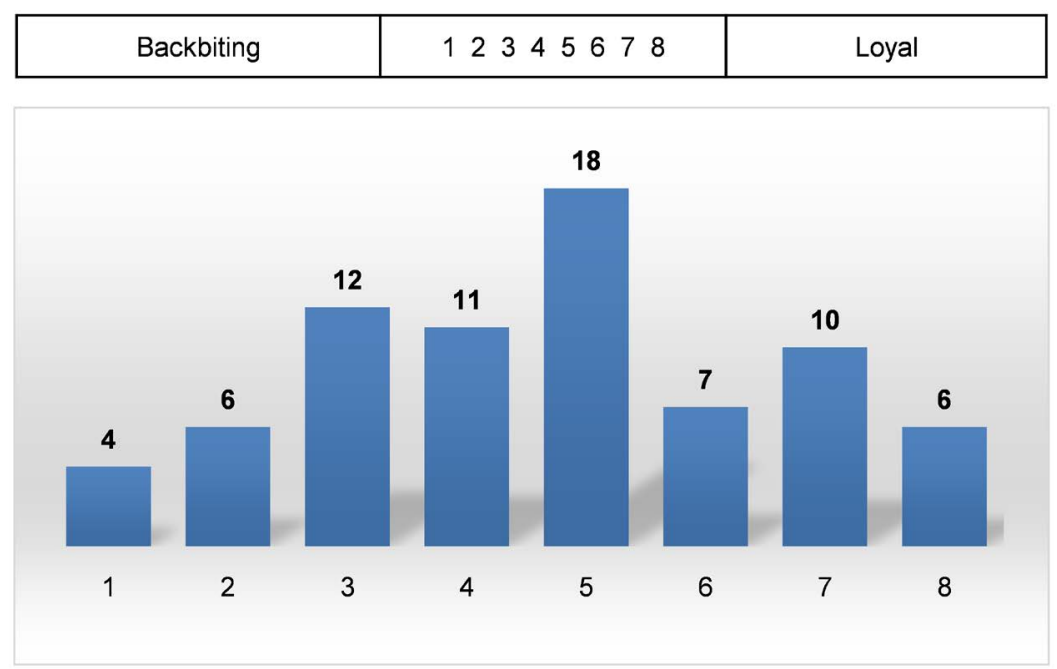

Figure 13. Presentation of the results for the twelfth question from the contingency approach questionnaire.
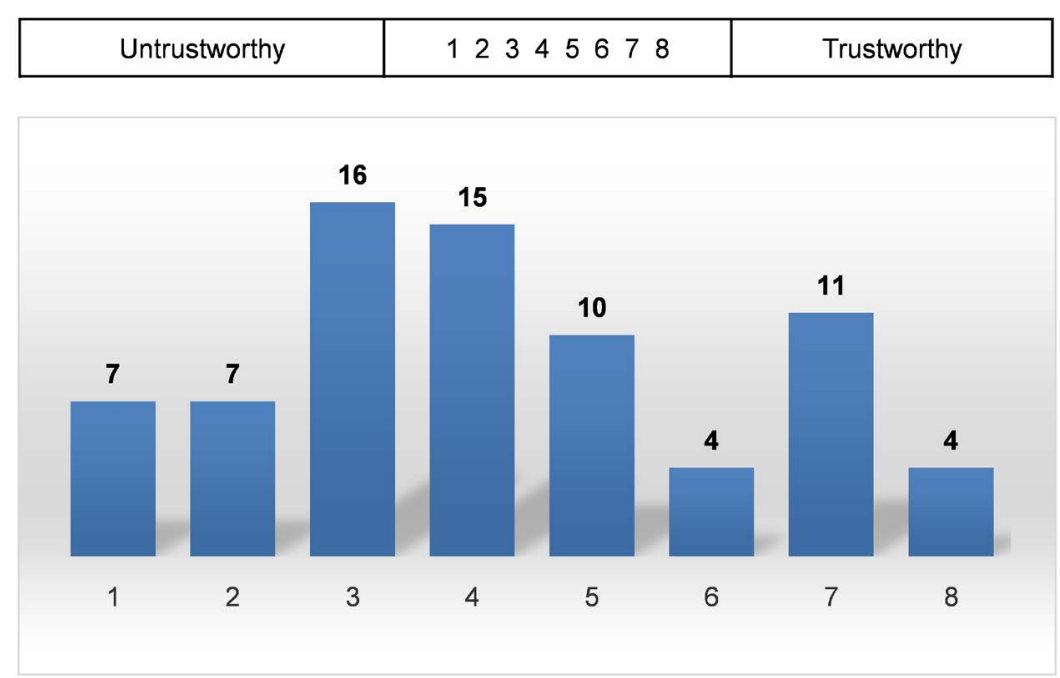

Figure 14. Presentation of the results for question thirteen from the contingency approach questionnaire. 
In the thirteenth question with 74 persons surveyed, there are 7 persons who have printed 1, 9.5\%, 7 persons who have printed 2, 9.5\%, 16 persons who have printed 3, 21.6\%, 15 persons who have printed 4, 20.3\%, 10 people who typed 5 so $13.5 \%$, 4 people who typed 6 so $5.4 \%, 11$ people who typed 7 so $14.9 \%$, and finally 4 people who typed 8 so $5.4 \%$. The largest number turns out to be the click of the number 3 , while the lowest click turns out to be the number 6 and 8 in this case.

In the fourteenth question with 74 respondents result in 4 persons who typed 1 so $5.4 \%, 6$ persons who typed 2 so $8.1 \%, 12$ persons who typed 3 so $16.2 \%, 11$ persons who typed 4 so $14.9 \%, 18$ people who typed 5 so $24.3 \%, 7$ people who typed 6 so $9.5 \%, 10$ people who typed 7 so $13.5 \%$, and finally 6 people who typed 8 so $8.1 \%$. Be the click of the number 5 , while the lowest click turns out to be the number 4 in this case.

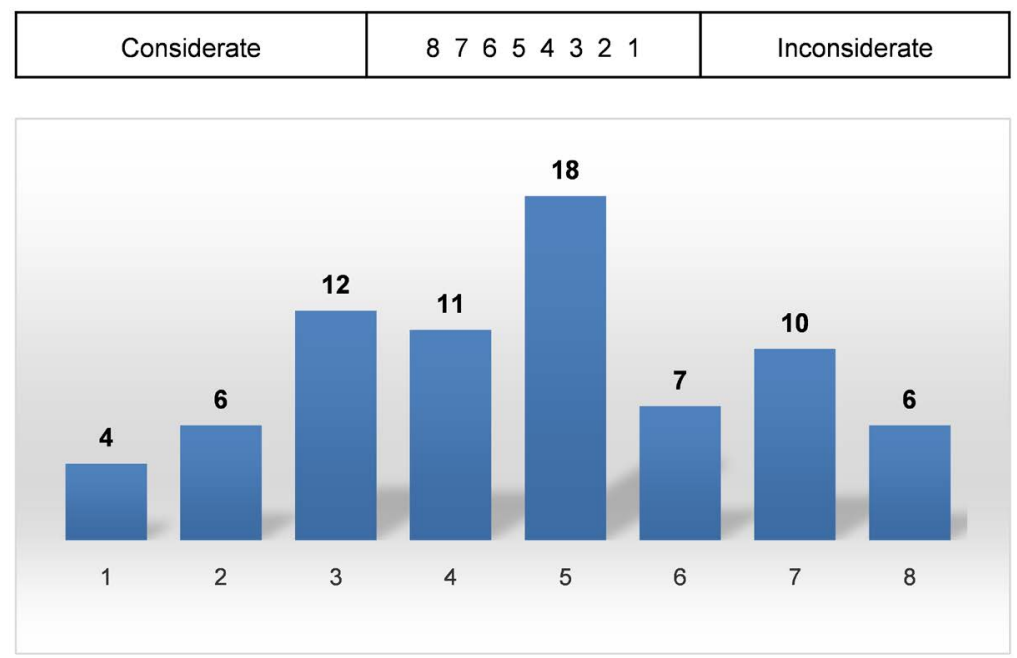

Figure 15. Presentation of results question fourteen from the contingency approach questionnaire.

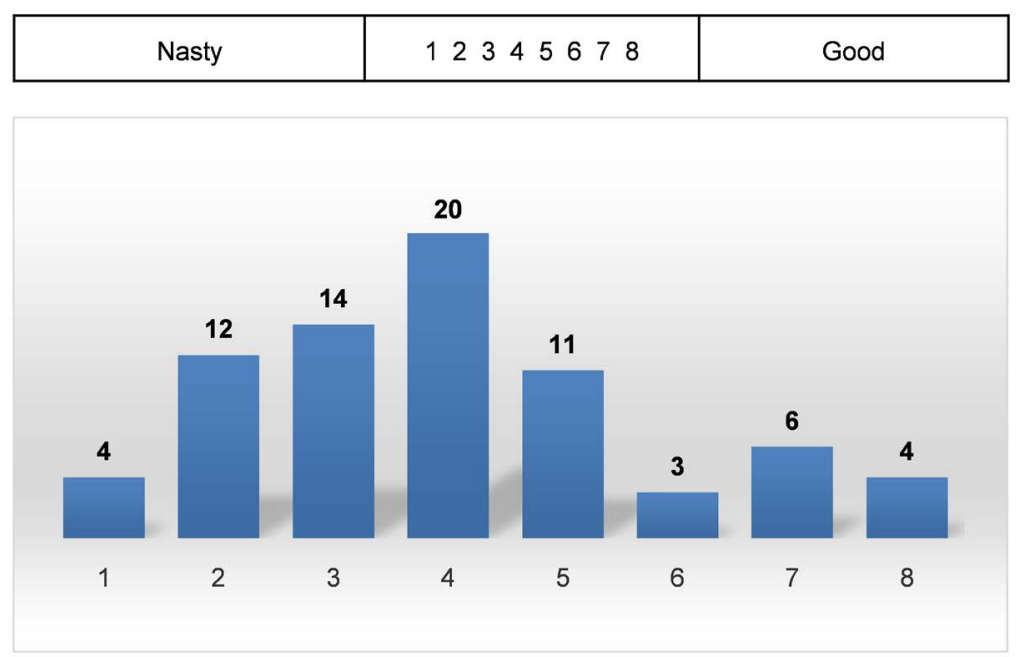

Figure 16. Presentation of results for question fifteen from the contingency approach questionnaire. 
In the fifteenth question with 74 respondents, there are 4 persons who have printed 1, 5.4\%, 12 persons who have printed 2, 16.2\%, 14 persons who have printed 3, 18.9\%, 20 persons who have printed 4, 27\%, 11 people who typed 5 so $14.9 \%$, 3 people who typed 6 so $4.1 \%$, 3 people who typed 7 so $8.1 \%$ and finally 4 people who typed 8 so $5.4 \%$. The largest number turns out to be the click of the number 4, while the lowest click turns out to be the number 6 in this case.

In the sixteenth question with 74 respondents result in 3 persons who typed 1 so $4.1 \%, 10$ persons who typed 2 so $13.5 \%, 13$ persons who typed 3 so $17.6 \%, 22$ persons who typed 4 so $29.7 \%$, 10 people who typed 5 so $6.8 \%, 7$ people who typed 7 so $9.5 \%$, and finally 4 people who typed 8 so $5.4 \%$. The largest number turns out to be the click of the number 4 , while the most click low turns out to be number 1 in this case.

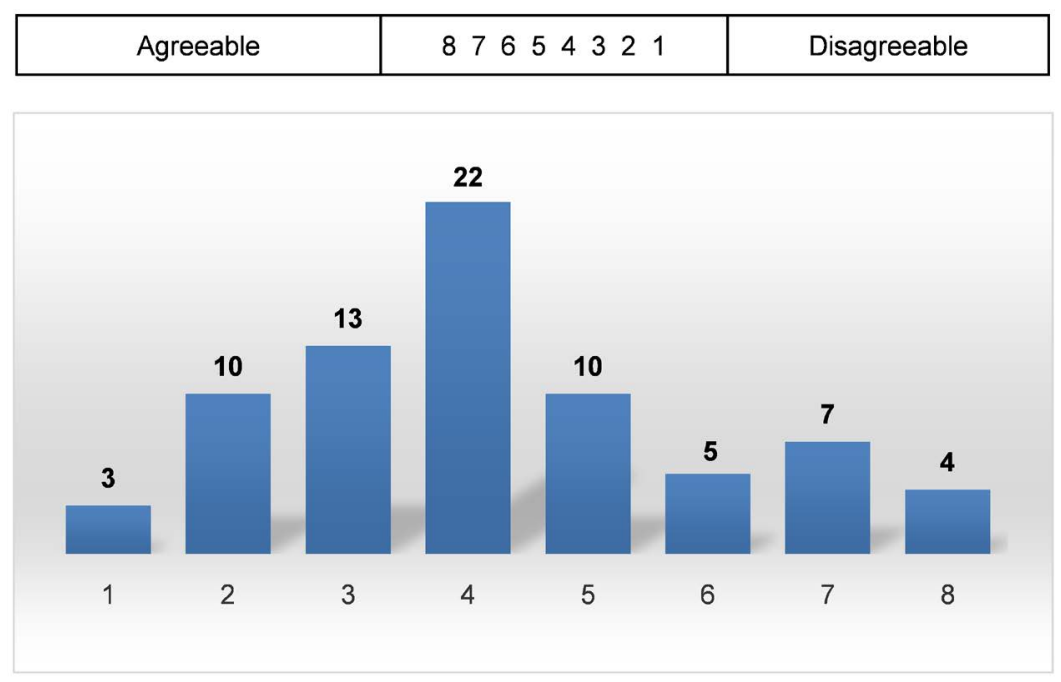

Figure 17. Presentation of results for question sixteen from the contingency approach questionnaire.

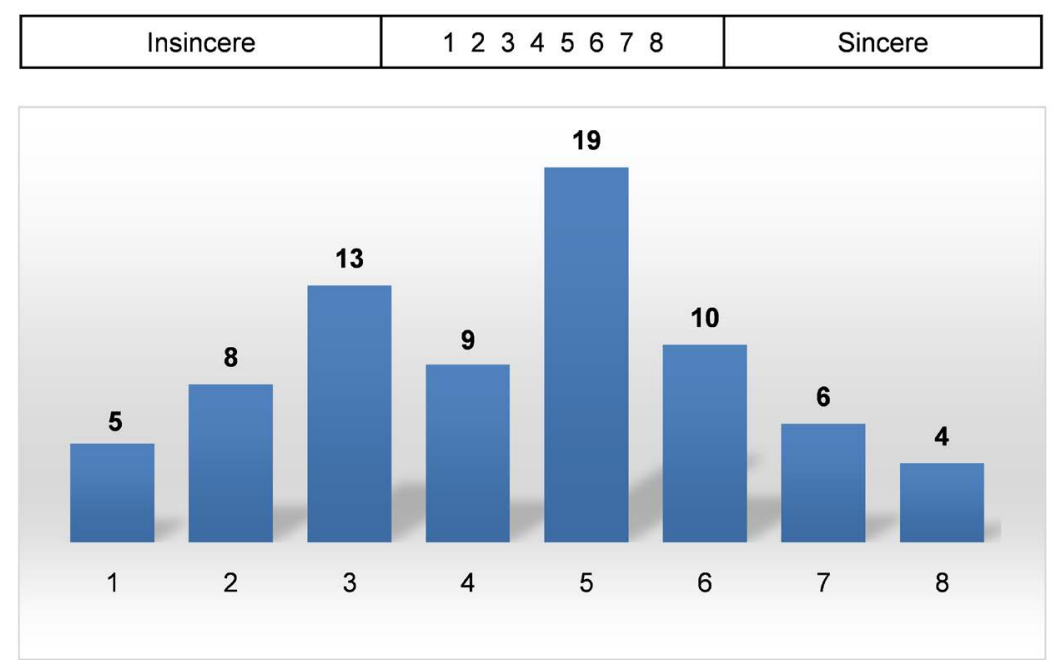

Figure 18. Presentation of the results for the seventeenth question from the access questionnaire Contingency. 
In the seventeenth question with 74 persons surveyed, there are 5 persons who have typed $1,6.8 \%, 8$ persons who have typed $2,10.8 \%, 13$ persons who have typed $3,17.6 \%, 9$ persons who have typed $4,12.2 \%, 19$ people who typed 5 so $25.7 \%, 10$ people who typed 6 so $13.5 \%, 6$ people who typed 7 so $8.1 \%$, and finally 4 people who typed 8 so $5.4 \%$. The largest number turns out to be be the click of the number 5 , while the lowest click turns out to be the number 8 in this case.

In the eighteenth question with 74 respondents, there are 4 persons who have printed 4, 5.4\%, 9 persons who have printed 2, $12.2 \%, 9$ persons who have printed 3,12.2\%, 12 persons who have printed 4, 16.2\%, 20 people who typed 5 so $27 \%, 8$ people who typed 6 so $10.8 \%, 7$ people who typed 7 so $9.5 \%, 5$ people who typed 8 so $6.8 \%$. The largest number turns out to be the number click 5 , while the lowest click turns out to be number 1 in this case.

It turns out that the participants have an average age of 24.5 years. The smallest number of respondents is among 18, persons who work in companies in a limited way. Then the second age group with more participants is $31-35$, then the age group 27 - 30 as the third in size and then 18-22, and the dominance of the age group in the questionnaire is $23-26$.

The smallest number of respondents is 2015 , then continuing with 2019 which justifies the beginning of the year to be a second smaller number of respondents who participated, continuing with 2016, and then 2018. The largest number of respondents is in 2017.

\subsection{Presentation of the Results Summarized by the Contingency Approach Questionnaire}

As shown in Figure 20, the result of the questionnaire turns out to be $43.7 \%$ with 64 points and more in the contingent access framework, 36.5\% with 57

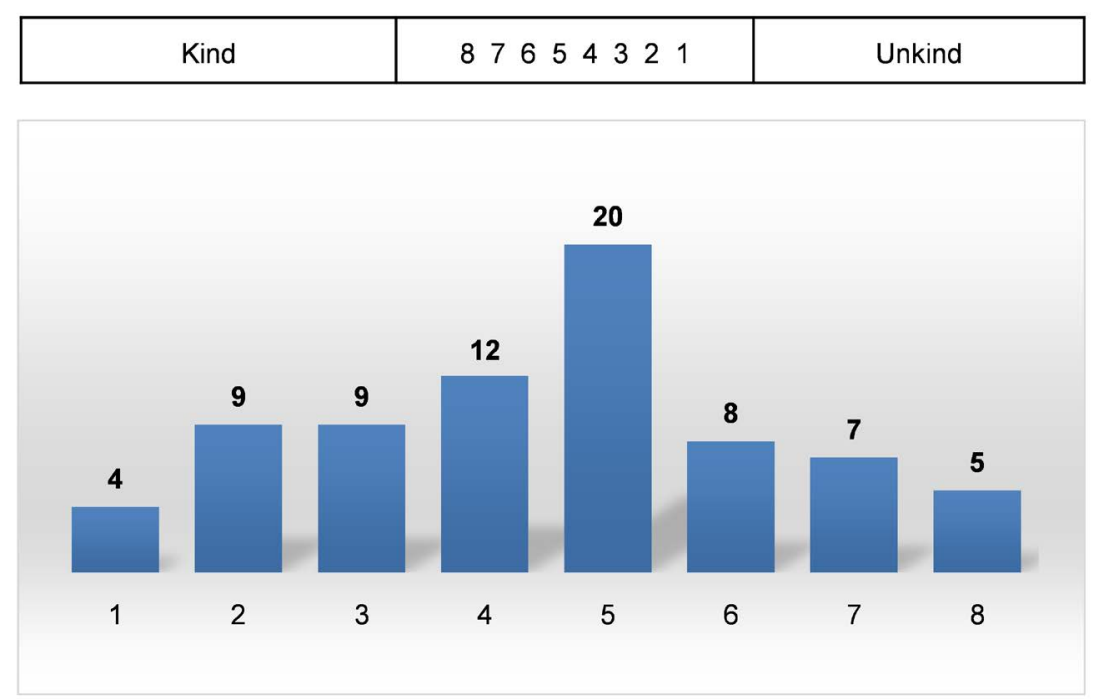

Figure 19. Presentation of the results for question eighteen from the contingency approach questionnaire. 


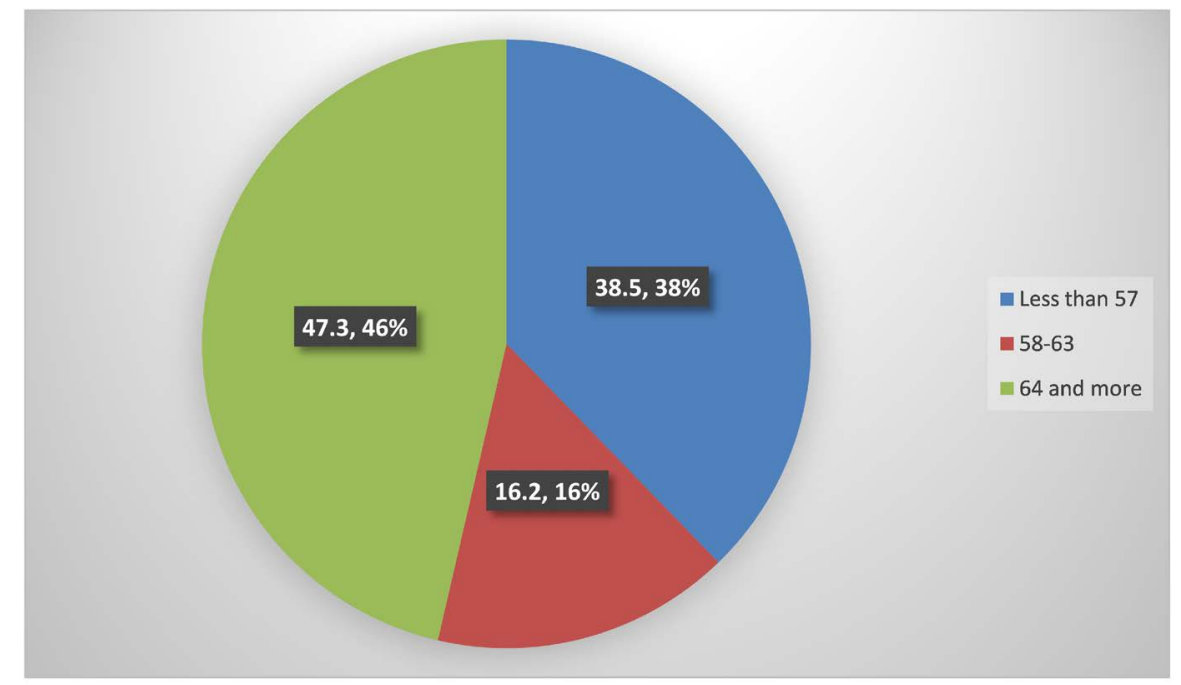

Figure 20. Presentation of results summarized from the contingency approach questionnaire.

points or less, and finally the result with the lowest number of persons belonging to this category is $16.2 \%$ between points $58-63$. From the persons interviewed in the two teams working in Pristina for the company Avrios it appears that the type of potential and existing Leaders with a higher percentage to be Leaders motivated by relationships with others. This is more than understandable since the Atmosphere in the Company is in the parts where these styles of leaders are best suited to the situations in the contingent approach, so in the pillars 4, 5, 6, 7 .

Avrios can be described as a company where the leader has an excellent relationship with the followers, the structure of Avrios Company is not very formal, and it is weaker if we compare it with a high structure such as Banks. The power or strength of the leader in can be described as weak or not very strong as there is leader with higher position in other parts.

Some of the problems we have encountered during interviews with potential people have been various mostly revolves around the thought of a person they preferred to work with the least, asking frequently asked questions what this means for my leadership style, or why do not think of a person I prefer to work with. My answer would be that you are objective in thinking about that person, and the emotions would be more measurable when you think of a person who prefers to work less. Then another problem was that sometimes it was difficult to distinguish a worker they did not like with a worker who preferred to work less. It is very difficult when the persons in question took family members as a less preferred person to work as in many cases there was no talk of any work done, but of personality discrepancies in general and not at the end of any work.

\section{Conclusions}

This research on the contingent approach and its impact on the company, especially for companies that use the contingent approach give many conclusions 
about identifying key personality factors of different employees, whose personality shows how to cope with different situations based on their way of thinking, i.e. their leadership style. There are factors that influence the identification of leadership styles. These factors should be analyzed by management, in order to create the possibility of intervention through work programs and action plans to increase the level of recognition as a factor of organizational success at work.

The results place a great deal of emphasis on the Leadership-Leadership League which is undoubtedly a strong factor that can identify a person what potential leadership style he has. This is emphasized when it is observed that some employees seek more approval from the team leader and also colleagues, while some others tend to appear less interested in some kind of approval or praise to the leader or colleagues.

Significant research result shows extremely great importance to the personality of a worker. This approach focuses on one person's attitude towards another person who can tell a lot about his or her personality. Since we know that personality tends not to change at least for a long time we can say that the results for each of the respondents do not have very large differences, which keeps the research usable for a long time.

The results of the research showed that job satisfaction greatly affects the effectiveness of the leader's work in placing him or her in the appropriate context.

\section{Recommendations}

The analysis of the results of the study through the developed questionnaire tends to give some recommendations that should be taken into account from the position of the leaders in the future, in order to increase the motivation for work among the employees, to develop the firm in general and above all. Increase productivity and enable its management within the firm.

In this regard, the following recommendations are listed:

- The recommendation is to increase engagement. Any person taking on a leadership role should commit to the group. The vision and mission of the group should be internal to the Leader. An effective leader is a person who can commit to using his or her ability to guide others, perform technical skills, and conceive of situations, thus helping to achieve the goal.

- The leadership style that a leader has a direct impact on the result achieved. This is what makes you memorable to others and fulfilled within yourself. Recommend that you know your style, are you a task-oriented leader, or more so from maintaining good relationships with colleagues.

- Leader positioning if done in time then, effectiveness can change the direction of the company, as the leader with the context adapted based on the style he has, results in effective and productive decision making.

- Recommend that when distributing the questionnaires, clarify about the person who is preferred to work the least. The reason is that a large proportion of interviewers have encountered dilemmas about this. The recommen- 
dation is to clarify this part of the questionnaire before distributing the questionnaires even if the instruction is next to the questionnaire.

The best leaders are human and socially aware. Recognizing the contribution of others and giving the team space to innovate is the best way to lead in a more sustainable future. Recommend that for jobs where communication is key to hire type two persons according to the contingent approach, while in places such as the sales world to hire a person of the first type.

\section{Conflicts of Interest}

The authors declare no conflicts of interest.

\section{References}

[1] Fiedler, F.E. and Chemers, M.M. (1974) Leadership and Effective Management. Scott, Foresman, Glenview.

[2] Fiedler, F.E. (1967) A Theory of Leadership Effectiveness. McGraw-Hill, New York.

[3] Fiedler, F.E. (1964) A Contingency Model of Leadership Effectiveness. In: Berkowitz, L., Ed., Advances in Experimental Social Psychology, Academic Press, New York, 149-190. https://doi.org/10.1016/S0065-2601(08)60051-9

[4] Fiedler, F.E. and Garcia, J.E. (1987) New Approaches to Leadership. Cognitive Resources and Organizational Performance. Wiley, New York. 